\title{
L'inclusion scolaire en France, un processus inachevé
}

Inclusive education in France, an unfinished process

La inclusión escolar en Francia, un proceso inacabado

\section{Alexandre Ployé}

\section{OpenEdition}

Journals

Édition électronique

URL : https://journals.openedition.org/ries/6618

DOI : $10.4000 /$ ries. 6618

ISSN : 2261-4265

\section{Éditeur}

France Education international

\section{Édition imprimée}

Date de publication : 1 septembre 2018

Pagination : 137-146

ISBN : 978-2-85420-619-7

ISSN : 1254-4590

Référence électronique

Alexandre Ployé, "L'inclusion scolaire en France, un processus inachevé », Revue internationale d'éducation de Sèvres [En ligne], 78 | septembre 2018, mis en ligne le 01 septembre 2020, consulté le 24 juin 2021. URL : http://journals.openedition.org/ries/6618 ; DOI : https://doi.org/10.4000/ries.6618 


\title{
L'inclusion scolaire en France, un processus inachevé
}

\author{
Alexandre Ployé \\ Université Paris-Est Créteil
}

On observe en France, depuis quelques années, une importante mutation des politiques scolaires, lesquelles, au gré des évolutions législatives concernant les personnes handicapées, ont pris un tournant plus inclusif. Ces évolutions concernent l'ensemble des apprenants que le ministère de l'éducation nationale nomme "élèves à besoins éducatifs particuliers ", parmi lesquels les élèves en grande difficulté scolaire, les élèves allophones, les élèves en situation de handicap, etc. Une telle mutation inclusive, dont maints auteurs s'accordent à penser qu'elle n'est pas achevée, repose sur de multiples reconfigurations du système éducatif (nouveaux dispositifs, nouvelles normes éducatives, promotion de nouvelles formes pédagogiques) mais également, en ce qui concerne spécifiquement le handicap, sur des assises philosophiques et anthropologiques renouvelées, qui permettent d'envisager, avec un regard neuf, la place qu'occupent les personnes handicapées dans notre société. Comment et dans quelles limites notre société soutient-elle sa mutation inclusive?

Cet article vise donc à établir un état des lieux fragmentaire de cette question, en privilégiant une entrée par le thème de l'inclusion scolaire des élèves en situation de handicap, en faisant l'hypothèse que cette problématique est métonymique de la question plus large de l'éducation inclusive et de l'inclusion sociale. L'école peut à bon droit être considérée comme un analyseur de la capacité de notre société à promouvoir un nouveau modèle qui affirme avec force le principe de non-discrimination entre les citoyens et la reconnaissance des droits des personnes handicapées. Les difficultés et les réussites de l'école française à modifier son regard sur ceux de ses élèves qui présentent des besoins éducatifs particuliers, à les prendre en compte dans des curricula pleinement inclusifs et à être un promoteur de leur participation pleine et entière à la vie sociale par l'accessibilisation des savoirs et la compensation de leurs difficultés, nous paraissent reproduire à l'échelle scolaire les progrès et butées de l'inclusion sociale, au sens large.

C'est pourquoi cet article, focalisé sur la question scolaire, se propose d'explorer celle-ci en quatre temps : nous montrerons d'abord comment, à l'échelle macro, le projet inclusif repose sur une nouvelle approche anthropologique de la personne handicapée et sur des visions concurrentes ou congruentes de l'inclusion scolaire. Puis, à l'échelle micro de la classe, nous questionnerons, par un détour clinique, comment s'ourle dans le quotidien de la classe la relation 
à l'élève handicapé, dans sa dimension psychique. Est-elle porteuse d'une créativité pédagogique renouvelée ou de résistances à la transformation ? Nous inférerons des deux premiers points des besoins de formation nécessaires au renforcement du pouvoir d'agir des enseignants, avant d'élargir le propos et de montrer que la réussite de l'inclusion scolaire est aussi déterminée par la capacité des professionnels de l'éducation et des parents des élèves en situation de handicap à créer de nouvelles formes de collaboration, dans le sens d'une «culture partagée» (Chauvière et Plaisance, 2008) de l'éducation inclusive.

\section{UN NOUVEAU REGARD SUR LA PERSONNE HANDICAPÉE}

Le syntagme "personne handicapée » est en usage depuis la loi de 1975, dite loi d'orientation en faveur des personnes handicapées ${ }^{1}$. Cette loi, pensée comme un tournant historique et préparée très en amont de sa parution, mobilise le terme handicap pour construire une catégorie à la fois synthétique - il inclut l'ensemble des populations autrefois désignées par l'infirmité, l'invalidité et l'ensemble des inadaptations psychiques et cognitives - et évolutive ; en effet le texte législatif se refuse à écrire une définition arrêtée de ce qu'est le handicap. Voulue précisément par S. Veil, qui défendit le projet de loi à l'Assemblée nationale, cette absence de définition est un premier pas vers une acception plus situationnelle du handicap, si bien que la loi laisse les commissions départementales qu'elle crée juger qui est ou n'est pas une personne handicapée. Notons également que cette loi pose un premier jalon dont l'éducation nationale s'empare lentement, celui de l'intégration scolaire. Ainsi, tout un imaginaire de la rupture avec ce qu'on se représente alors comme une période de ségrégation des personnes handicapées préside à l'écriture de ce texte fondateur.

Le syntagme "personne handicapée » est également inscrit dans le marbre des législations les plus récentes, comme la loi du 11 février 2005 «pour l'égalité des droits et des chances, la participation et la citoyenneté des personnes handicapées $^{2}$ » qui institue des maisons départementales chargées d'établir le projet de vie et les moyens à mettre en œuvre en vue de sa réalisation pour chaque personne qui justifierait d'une situation de handicap. Ce renouvellement législatif de 2005 est particulièrement fécond pour le champ de l'éducation inclusive, puisqu'il porte avec lui deux notions connaissant immédiatement des traductions pédagogiques : la compensation et l'accessibilité, qui, dans l'agir enseignant, peuvent être pensées au travers de l'adaptation et de la différenciation pédagogique. Cette nouvelle loi provoque donc à la fois un ressourcement du rôle des enseignants, que l'on peut ainsi considérer comme ayant le pouvoir de réduire

1. Loi $n^{\circ} 75-534$ du 30 juin 1975 d'orientation en faveur des personnes handicapées.

2. Loi $n^{\circ}$ 2005-102 du 11 février 2005 pour l'égalité des droits et des chances, la participation et la citoyenneté des personnes handicapées. 
des situations de handicap ${ }^{3}$ à l'école, du fait de leur expertise pédagogique, et une réécriture de certains textes organisant les dispositifs de l'enseignement spécialisé. Ainsi donc, de manière peut-être davantage symbolique qu'organisationnelle, les " unités pédagogiques d'intégration », au collège, deviennent, à la rentrée de 2010, des " unités localisées pour l'inclusion scolaire" (ULIS). L'inclusion, plutôt que l'intégration. La Loi de refondation de l'école de la République (2013) entérine le changement et promeut l'inclusion scolaire comme grand axe des politiques éducatives. Notons à ce propos que la notion d'inclusion dépasse alors la seule scolarisation des élèves en situation de handicap. Le terme englobe toutes les formes d'altérité à l'école, comme les élèves allophones ou encore les élèves à haut potentiel...

Au-delà de ces aspects législatifs fondamentaux, nous avons choisi mettre ici l'accent sur les modifications anthropologiques qui permettent de penser le modèle de société inclusive, dont l'inclusion scolaire est un pilier essentiel. En effet, les travaux de Stiker (2005) et de Gardou (2012), après ceux de Foucault dans son Histoire de la folie à l'âge classique (1961), rappellent combien les personnes handicapées ont été longtemps frappées par un réflexe social d'exclusion, de création de lieux dédiés, en marge de la société commune, sur le modèle de ceux que Foucault décrit : l'hôpital général au XVII ${ }^{\mathrm{e}}$ siècle, censé rassembler en un lieu clos et à l'écart toutes les dimensions de la marginalité urbaine de l'époque, et l'asile de Pinel, au XIX ${ }^{e}$ siècle, qui, sous couvert d'un projet de soin "révolutionnaire », enferme les fous dans des structures spécifiques. Gardou (2012) rappelle que cette histoire fabrique

des êtres atopos, sans place dans la société. Expropriées [les personnes en situation de handicap], maintenues dans des hors lieux. [...] Rendues invisibles, ontologiquement gommées.

L'éloignement des infirmes et des fous, historiquement, a pu constituer une première manière de circonscrire l'angoisse anthropologique liée à leur étrangeté. Il fallait les soustraire à notre vue, les reclure en quelque endroit point trop éloigné mais à l'abri de notre regard, seuls quelques pédagogues comme Valentin Haüy (1745-1822), avec les aveugles, l'abbé de l'Épée (1712-1789), avec les sourds, et le médecin Jean-Gaspard Itard (1774-1838), avec Victor, l'enfant sauvage de l'Aveyron, s'essayant à réinscrire les handicapés parmi les figures d'une humanité diverse, avec plus ou moins de bonheur. À quelle révolution du regard la transition vers une société inclusive appelle-t-elle? Gardou (ibid.) en résume ainsi les enjeux :

Au rebours d'une logique disjonctive, fondée sur une conformité fantasmatique, l'optique inclusive se caractérise par la capacité collective à conjuguer les singularités, sans les essentialiser.

3. L'expression « situation de handicap ", d'usage fréquent à l'éducation nationale, est une sorte de dépassement de la loi de 2005, en ce sens qu'elle donne au terme handicap une acception pleinement sociale, tandis que l'article 1 de la loi de 2005 se tient dans un entre-deux : le handicap y est certes perçu de manière sociale, comme restriction de participation, mais issu d'une déficience, qui maintient une influence biomédicale dans cet effort de définition. 4. Loi $n^{\circ}$ 2013-595 du 8 juillet 2013 d'orientation et de programmation pour la refondation de l'École de la République. 
Ainsi, la société inclusive est celle qui se départit de la tyrannie du même. Elle consiste pour l'Homme à rompre avec les processus d'altérisation qui ont dominé, jusqu'à récemment, les rapports de chacun avec ses semblables; rompre avec la marginalisation, la construction de stratagèmes de surveillance, de punition et d'exclusion de celui qui menace le sentiment de l'Un, celui qui ne remplit pas son « devoir de conformité » (ibid). Il s'agit donc d'inventer une norme inclusive fondée sur l'idée que le commun de l'humanité s'origine autour de sa diversité et de sa vulnérabilité. La société inclusive - et donc l'école inclusive - est celle dans laquelle les sujets échangent de l'adaptation réciproque. Une question se pose alors : de quand daterait en France cette révolution, si l'on tient pour acquis qu'elle a déjà débuté ? Des lois récentes sur le handicap et l'école inclusive?

Avec Swain et Gauchet (1994), nous partageons ici le sentiment que, contrairement à ce qu'avancent Foucault et les foucaldiens, la révolution a commencé... avec la Révolution de 1789. Réinterprétant le geste pinélien, c'est-à-dire le mythique désenchaînement des fous et l'invention de l'asile, Swain propose une thèse que nous pensons féconde pour penser les ambiguïtés du système inclusif français actuel : finalement, que l'asile soit un lieu « autre ", une clôture, qu'il construise l'enfermement des fous entre quatre murs et sous la surveillance d'un nouveau pouvoir relais de l'État, celui des médecins, c'est un fait. Mais, pour Swain, l'important est davantage à saisir dans l'entreprise de soins prenant appui sur la parole entamée par Pinel, dont toute la thérapeutique vient dire que le fou n'est pas tout entier sujet de sa folie, qu'il demeure en lui l'homme de raison, celui du XVIII ${ }^{\mathrm{e}}$ siècle et des Lumières; homme de raison, en partie aliénée, avec lequel une circulation de la parole est non seulement possible, mais posée par ce médecin aliéniste comme un acte de soin. "Passion démocratique ", l'inclusion, dit Swain (1994), utilisant ce mot bien avant ses modernes thuriféraires, c'est non pas errer dans la cité dans l'indifférence comme un animal, mais être ré-inscrit dans la circularité de la parole, fût-ce à l'asile. L'exclusion des fous, comme celle des infirmes, était «de ne pas appartenir au cercle de l'humanité défini par la communication".

Ainsi, nous ne pouvons réduire la question de l'inclusion scolaire à celle du partage d'un lieu unique et commun qui serait la classe ordinaire. Les enseignants spécialisés, fondant leur action sur le credo de l'éducabilité, et travaillant au sein d'institutions qui, pour être éloignées de l'école, n'en sont pas pour autant des asiles, n'œuvrent-il pas dans le quotidien de leurs échanges pédagogiques, à l'inclusion de leurs élèves dans le cercle commun de l'humanité ? L'inclusion en France repose donc sur un aggiornamento anthropologique qui marche sur deux jambes théoriques: la première propose de penser qu'il faut que le système inclusif s'étaye sur une nouvelle norme de diversité et de vulnérabilité qui fonde l'humanité et son destin commun, la seconde avance que le partage de lieux communs ne suffit pas à faire l'inclusion, mais que celle-ci se définit avant tout par la capacité à inscrire le sujet handicapé dans la circularité d'une communication inter-humaine. 


\section{LES ENSEIGNANTS ET LES ÉLÈVES HANDICAPÉS DANS LE QUOTIDIEN DE LA CLASSE ORDINAIRE}

Envisageons désormais la mutation inclusive à l'échelle micro de la classe et des pratiques enseignante. De nombreuses recherches, aussi bien en sociologie de l'éducation qu'en didactique, soulignent que les enseignants incluant dans leurs classes des élèves en situation de handicap sont souvent en difficulté, quand il s'agit d'articuler les savoirs à transmettre et les besoins d'apprentissage spécifiques de ces élèves. L'existence de "contrats didactiques différentiels " (Suau ; Assude, 2016), les pratiques de différenciation pédagogique et d'adaptation des contenus ou de supports de cours sont, dans la classe, des vecteurs d'accessibilité didactique mais peuvent également y faire obstacle, en ce sens que l'élève inclus peut devenir l'objet de visées particularisantes qui diminuent la dimension inclusive de sa présence en classe.

[Il faudrait] parvenir à ne plus opposer différenciation/individualisation/ adaptation, mais envisager plutôt le continuum qui les lie, amène à repérer la complexité de la conception de l'adaptation des enseignements en contexte de classe ordinaire. (Gombert et al., 2017)

Cette étude récente de Gombert et al. conclut à la nécessité d'une attention et d'ajustements permanents aux besoins des élèves, une créativité pédagogique dont il n'est pas toujours aisé de faire preuve.

Nous voudrions à ce sujet mettre ici l'accent sur des limitations aux pratiques d'inclusion, dont les causalités ne renvoient pas immédiatement à un défaut de maîtrise pédagogique et didactique mais plutôt à des éprouvés psychiques spécifiques attachés à la rencontre dans la classe avec un élève handicapé. Nous avons ainsi pu montrer (Ployé, 2016) qu'à la réussite de l'inclusion scolaire correspond la plus ou moins grande capacité des enseignant à intégrer psychiquement le sentiment d'inquiétante étrangeté ( «unheimlich», Freud, 1909) que provoque la rencontre avec le handicap, notamment mental. Ces élèves inquiètent au double titre de leur caractère d'altérité et de familiarité : marqués par la vulnérabilité, le manque rendu visible par le handicap, ils sont l'image de la vulnérabilité et du manque propres à l'humanité toute entière. Ils sont le miroir dans lequel nous nous découvrons "étrangers à nous-mêmes ", c'est-à-dire toujours limités dans la connaissance de notre propre inconscient. Leur altérité est possiblement la nôtre. Au contact de ces élèves, les enseignants peuvent éprouver des affects violents : au premier rang de ces affects, nous avons repéré la honte et la culpabilité. Le sentiment de ne pas être à la hauteur de l'idéal inclusif, partagé par une grande partie des professionnels, provoque également de vifs moments de désarroi professionnel, lequel peut inaugurer des mouvements de retrait ou de "diminution-restriction" (Simon, 1999) de l'agir enseignant. Nous proposons donc de penser que la situation inclusive est susceptible 
de produire les conditions d'une vulnérabilisation identitaire des enseignants, en écho à la vulnérabilité des élèves handicapés. Pour en atténuer le choc, on observe alors parfois, chez les professeurs, la mobilisation de stratégies défensives, qui consistent à expulser du champ pédagogique l'élève handicapé perçu comme persécuteur (on le refuse en classe ou bien on le cantonne à des tâches secondes) ou encore à l'invisibiliser (les élèves ne sont plus regardés, quoique présents).

À ces mouvements psychiques puissants correspondent des formes dégradées de l'agir pédagogique (Ployé, 2013) : on peut en effet parfois observer que la situation inclusive est hybridée par des habitudes intégratives, dans lesquelles les élèves inclus ne le sont plus qu'au titre de leur socialisation. Nous avons in fine désigné par le mot d'euphémisation ces stratégies conscientes et inconscientes, qui réduisent l'impact de la mutation inclusive. Elles aboutissent au total au maintien des élèves en situation de handicap dans une position d'entre-deux, une position de seuil restant à franchir, une liminalité qui les définit par une double négation : ils ne sont ni parfaitement inclus ni réellement exclus Cette position sociale de liminalité résiste au changement et s'inscrit dans le temps long. Cette constatation conduit à penser que l'inclusion, en France, demeure un processus pour le moins inachevé.

\section{DES BESOINS DE FORMATION POUR RÉUSSIR LA MUTATION INCLUSIVE}

La naissance d'un nouveau cadre pour la formation des enseignants spécialisés en 2016, le CAPPEI ${ }^{5}$, montre combien le processus est en effet inachevé, puisqu'il faut le relancer à l'Éducation nationale par une nouvelle formation, laquelle s'adresse à l'ensemble des enseignants qui désirent se spécialiser ; cette formation témoigne d'une nouvelle conception de l'enseignement spécialisé marquée par le recul des options fondées sur une approche médicale du handicap, par le développement d'un large tronc commun de formation destinés à tous les enseignants spécialisés des deux degrés et par une approche différenciée par type de poste occupé par les professionnels. Ce nouveau cadre nous paraît l'occasion de traduire certains contenus de formations en suivant trois directions qui nous semblent répondre aux besoins de tous les enseignants, tels que nous pouvons les déduire des difficultés constatées plus haut.

La première est celle d'une invitation à une réflexivité éthique. Afin que la prescription à l'inclusion ne soit pas vécue par les professionnels sur le mode d'un idéal impossible à atteindre ou comme l'ordre d'un surmoi institutionnel tyrannique, il convient de donner aux professionnels, ceux-là mêmes qui constituent le front pionner de la mutation inclusive, les moyens d'élaborer pour euxmêmes et par eux-mêmes une position éthique incarnée et non subie. Nous

5. Certificat d'aptitude professionnelle aux pratiques de l'éducation inclusive, Bulletin officiel $n^{\circ} 7$ du 16 février 2017. 
faisons le choix, pour notre part, de mobiliser le détour de l'histoire et du questionnement socio-historique pour inviter les stagiaires à penser des dilemmes professionnels. Ces dilemmes portent de manière sous-jacente les tensions du développement français de l'éducation inclusive : comment concilier la volonté inclusive et l'existence de dispositifs spécifiques ? Nous invitons les stagiaires à se situer d'abord par rapport à un débat qui a marqué la naissance de l'enseignement spécialisé : la naissance, en 1909, des classes de perfectionnement est-elle l'indice que le système éducatif français décide de scolariser les enfants handicapés par un détour ségrégatif ou bien ces classes de perfectionnement témoignent-elle de l'affirmation par l'État d'une éducabilité généralisée, au-delà des déficiences individuelles ? Nous prolongeons le débat en lui donnant la dimension de la controverse philosophico-historique qui oppose, d'un côté, Foucault et ceux qu'il a inspirés et, de l'autre Swain et Gauchet, telle que présentée en début d'article. Adopter une posture réflexive sur des définitions apparemment opposées de l'inclusion permet de construire un jugement professionnel auto et hétéro-référencé avec lequel il est plus simple de négocier qu'avec une prescription institutionnelle qui demeurerait insuffisamment pensée par ceux qui sont chargés de la faire entrer en vigueur.

Le second axe est l'analyse clinique de la pratique professionnelle, dont nous pensons qu'elle est un dispositif propre à aider les enseignants à élaborer leur conflictualité interne dans leur rapport aux élèves handicapés. La formation des enseignants ne saurait s'en tenir à une approche technicienne ou à une logique instrumentale. Elle devrait également faire place à un travail de ré-implication subjective de la position professionnelle en contexte de grande hétérogénéité. Ainsi, une approche clinique me paraît favorable à la reprise d'un processus d'appropriation subjective de la conflictualité à l'œuvre chez les enseignants, dans la rencontre avec les élèves. Il s'agirait d'y rompre avec l'illusion d'une totale maîtrise de soi pour développer quelque tolérance à l'endroit du jeu des instances psychiques. Il s'agirait également d'y favoriser, par l'écoute clinique groupale ${ }^{6}$, une meilleure intégration des instances psychiques du sujet professionnel afin que la scène scolaire ou professionnelle ne soit plus le lieu où s'actualisent des conflits infiniment répétés et infiniment refoulés. C'est, selon nous, la condition d'une capacité plus grande à tolérer l'autre et son inquiétante étrangeté.

Enfin, un troisième axe serait de généraliser les attendus des formations spécialisées à la formation initiale des enseignants. Si le référentiel de compétences des enseignants mentionne que ceux-ci doivent savoir " prendre en compte la diversité des élèves ${ }^{7}$, il nous semble que l'approche formative du master Métiers de l'enseignement, de l'éducation et de la formation (MEEF) demeure

6. Nous faisons allusion ici aux dispositifs d'analyse clinique de la pratique professionnelle inspirés des travaux du psychanalyste britannique Balint qui, après-guerre, invitait des médecins sous sa supervision à travailler en groupe leur rapport subjectif aux malades, à la maladie, à la mort.

7. Le référentiel de compétences des métiers du professorat et de l'éducation. [https://goo.gl/vQwXjZ] 
largement disciplinaire : l'entrée s'y fait par la didactique des disciplines à transmettre plutôt que par la prise en compte des besoins des élèves. A minima, une formation à l'école inclusive devrait promouvoir une articulation des deux entrées : par la didactique et par les besoins.

\section{De nOUVELles fORMES DE COLLABORATION POUR RÉUSSIR L'ÉDUCATION INCLUSIVE}

Faire reposer la réussite du tournant inclusif sur les seules épaules des enseignants serait une erreur. Il convient d'adopter une logique intégrative de prise en compte, sur un processus commun, le développement de l'éducation inclusive, des compétences diverses qui traversent l'ensemble des partenaires et leurs territoires respectifs : enseignants, accompagnants des élèves en situation de handicap (AESH), éducateurs et parents. Si les réunions d'équipe de suivi (REE) et autres équipes de suivi de scolarisation (ESS) des élèves handicapés comportent déjà cette dimension pluri-partenariale, nous pensons qu'une "culture partagée » de l'inclusion (Chauvière et Plaisance, 2008) reste à construire. Nous devons tendre vers une réelle « communauté éducative élargie et inclusive » (ibid.) qui se heurte, pour l'heure, à de nombreux obstacles, notamment quand on évoque le rôle et la place des parents de l'enfant en situation de handicap... Historiquement, la collaboration entre professionnels et parents autour de la question du handicap a pu pâtir des représentations extrêmement négatives véhiculées par certaines cultures professionnelles. Donnons-en deux exemples : en Grande-Bretagne, dans sa contestation radicale des cadres de pensée de la schizophrénie, Cooper, père fondateur avec Laing de l'antipsychiatrie britannique, rendait la famille directement responsable de la survenue de la maladie chez le jeune adulte, qui trouvait ainsi à se défendre contre l'oppression familiale ; dans le même temps, en France, toute une rhétorique de la mère froide, plus ou moins initiée par le découvreur de l'autisme, Léo Kanner, envahissait le champ d'une certaine littérature psychanalytique de mauvais aloi, qui aboutissait à recommander une stricte séparation des enfants autistes d'avec leur famille. On sait combien ce legs historique pèse encore sur la qualité des relations parents/soignants dans la prise en charge des troubles du spectre autistique. À rebours, les programmes éducatifs d'orientation comportementaliste ont posé comme principe le fait que, loin de lui nuire, les parents devaient être considérés comme les premiers spécialistes de leur enfant. La collaboration plutôt que l'affrontement conditionne la réussite de l'éducation inclusive. Nous proposons de suivre R. Scelles (2013), quand elle décrit comment les professionnels, du soin ou de l'éducation, doivent créer des alliances avec les parents : ils doivent d'abord proposer des aides «que la famille peut et veut accepter à ce moment-là de son cheminement »; les familles doivent se sentir "suffisamment valables pour être aidées»(ibid.). Les compétences des 
professionnels ne doivent pas être vécues comme le prétexte du discrédit des familles. Au contraire, les parents doivent se voir donner le temps « de construire leur propre savoir-être et savoir-faire avec leur enfant»(ibid.), débarrassés du regard évaluateur des professionnels. Cela sous-entend que lesdits professionnels aient au préalable pu élaborer la trame fantasmatique sur laquelle se tissent leur relation aux parents, car « entre parents et professionnels, des malentendus peuvent s'installer du fait de ces représentations imaginaires différentes entre eux » (Boissel, 2008). Ajoutons, pour conclure, que les professionnels doivent également développer la capacité à être aidés par les parents : ce sont les aides réciproques, les discours partagés, l'oblitération des frontières davantage fantasmatiques que réelles qui seront les vecteurs d'une mutation inclusive !

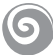

Cet article visait à un état des lieux de la question de l'inclusion scolaire en France, en tant qu'elle est un indicateur puissant de la transformation de la société française en un modèle plus inclusif.

Nous avons établi que cette mutation inclusive pouvait s'appuyer, au niveau macro, sur une perspective anthropologique renouvelée qui établit notamment, comme le dit C. Gardou, qu' "il n'y a pas de vie minuscule », et que nous devons construire une société " maison commune » qui admettrait «que chacun est légataire de ce que la société a de plus précieux; que l'humanité est une infinité de configurations de vie et une mosaïque d'étrangetés » (Gardou, 2012).

Mais nous avons également montré, à l'échelle micro de la classe combien ces étrangetés, à la fois familières et inquiétantes, pour reprendre la traduction usuelle de l'allemand « unheimlich» mobilisé par Freud pour décrire le sentiment qu'on peut éprouver à côtoyer notamment la folie, pouvaient agir négativement sur la capacité des enseignants à renouveler leur pédagogie et leur didactique dans le sens de ce qu'il est désormais convenu d'appeler l'éducation inclusive. Cette expression est un marqueur de plus d'un changement : nous ne parlons plus d'éducation spéciale, comme pendant la première moitié du $\mathrm{XX}^{\mathrm{e}}$ siècle, nous ne parlerons bientôt plus d'enseignement spécialisé, car l'expression « éducation inclusive » inclura la capacité du système éducatif dans son ensemble et de la variété de ces acteurs - élèves, accompagnants, enseignants et parents - à produire des adaptations réciproques. Mais l'usage de cette expression nouvelle n'a de valeur performative que limitée : elle impulse un changement réel mais ne doit pas masquer que nous sommes au milieu du gué, que les courants ne sont pas unanimes à nous porter sur la rive inclusive et que, dès lors, il faut prendre en compte les obstacles rencontrés, si nous ne voulons pas que le gué soit un seuil infranchissable. 


\section{BibLIOGRAPHIE}

BOISSEL A. (2008) : "Parentalité et handicap ", La lettre de l'enfance et de l'adolescence, $\mathrm{n}^{\circ} 73$, p. 63-70.

CHAUVIERE M., PLAISANCE É. (2008) : "Les conditions d'une culture partagée ", Reliance, $\mathrm{n}^{\circ}$ 27, p. 31-44.

GARDOU Ch. (2012) : La société inclusive, parlons-en!, Toulouse, Erès.

GOMBERT A. et al. (2017) : "Processus d'adaptation de l'enseignement en contexte inclusif: étude de cas pour un élève avec autisme ", Carrefours de l'éducation, $\mathrm{n}^{\circ} 43$, p. 11-25.

PLOYÉ A. (2013) : "Collaborer à des démarches d'inclusion au collège: analyse clinique des modalités pédagogiques et des éprouvés psychiques des acteurs", La Nouvelle revue de l'adaptation et de la scolarisation, $\mathrm{n}^{\circ}$ 61, p. 23-36.

PLOYÉ A. (2016) : Les enseignants aux prises avec l'étrangeté: approche clinique de l'inclusion des élèves handicapés au collège, Université Paris 8 Saint-Denis, thèse de doctorat sous la direction de Laurence Gavarini.

SCELLES R. (2013) : "Faire des membres de la famille des acteurs et des auteurs de leur devenir », Contraste, n $^{\circ} 37$, p. 223-243.

STIKER H.-J. (2005; 1982) : Corps infirmes et sociétés, Paris, Dunod.

SUAU G., ASSUDE T. (2016) : "Pratiques inclusives en milieu ordinaire : accessibilité didactique et régulations "; Carrefours de l'éducation, n 42, p. 155-159.

SWAIN G. (1994) : "Une logique de l'inclusion », in GAUCHET M., SWAIN G., Dialogue avec l'insensé, Paris, Gallimard, p. 112-130. 